\title{
The Primate Retina Contains Distinct Types of Y-Like Ganglion Cells
}

\author{
Ari Rosenberg ${ }^{1}$ and Vargha Talebi ${ }^{2}$ \\ ${ }^{1}$ Committee on Computational Neuroscience, University of Chicago, Chicago, Illinois 60637, and ${ }^{2}$ McGill Vision Research, McGill University, Montreal, \\ Quebec, Canada H3A 1A1
}

Review of Crook et al. (http://www.jneurosci.org/cgi/content/full/28/44/11277)

Review of Crook et al. (http://www.jneurosci.org/cgi/content/full/28/48/12654)

Our understanding of visual processing arises from studies conducted in different species. As such, establishing the extent to which the mechanisms encoding a visual scene are homologous across these species is important. This is perhaps most easily accomplished in the retina, where structure and function are closely linked.

The retina is comprised of a number of different circuits with distinct functions. Some circuits, such as the rod pathway important for seeing in low-light conditions, are highly conserved across mammals. Other circuits, like those underlying trichromatic color processing, are found in only a minority of species. Homologies between retinal ganglion cells (RGCs), the output units of the retina, are of particular interest because their responses reveal how a visual scene is transformed by the eye.

The Y-cell $(\alpha$-cell) first identified in the cat and morphologically conserved across many mammals stands out because of its unique physiological properties (Enroth-Cugell and Robson, 1966; Stone, 1983; Peichl et al., 1987). The existence of

Received Jan. 26, 2009; revised March 3, 2009; accepted March 9, 2009.

This work was supported by Department of Homeland Security Dissertation Grant DE-AC05-060R23100 (A.R.) and Natural Sciences and Engineering Research Council of Canada Postgraduate Scholarship D2-3489492007 (V.T.). We thank Jonathan B. Demb, Naoum P. Issa, and Felecia N. Rosenberg for their helpful comments and suggestions.

Correspondence should be addressed to Ari Rosenberg, Committee on Computational Neuroscience, University of Chicago, 947 East 58th Street, MC0926, Chicago, IL 60637. E-mail: arirose@uchicago.edu.

D0I:10.1523/JNEUROSCI.0423-09.2009

Copyright $\odot$ 2009 Society for Neuroscience $\quad$ 0270-6474/09/295048-03\$15.00/0 a primate homolog to the $\mathrm{Y}$-cell has been a controversial subject, but recent studies have conclusively identified at least two types of Y-like primate RGCs (Petrusca et al., 2007; Crook et al., 2008a,b), underscoring the need to incorporate Y-cell physiological properties into our understanding of visual processing.

Retinal cell types are defined by their morphological, anatomical, and physiological properties. Morphologically, Y-cells have large somas, thick axons, and wide dendritic fields. Anatomically, they project to both the lateral geniculate nucleus (LGN) and the superior colliculus. Physiologically, Y-cell receptive fields have a center-surround organization that is either ON- or OFF-centered, responding, respectively, to either step increases or decreases in light level (Stone, 1983). The dendrites of ON and OFF Y-cells stratify in separate levels toward the center of the inner plexiform layer (IPL), where they receive input from bipolar cells (Fig. $1 A)$. Depending on the stimulus, Y-cells respond either linearly or nonlinearly. Responses to drifting sinusoidal gratings (repeating patterns of light and dark stripes) are linear, modulating at the drift frequency of the stimulus (Fig. $1 \mathrm{Bi}$ ). Their wide dendritic (receptive) fields make Y-cells maximally responsive to low spatial frequency gratings (those with wide stripes) (Fig. 1C). Responses to high spatial frequency (thin striped) contrastreversing gratings (gratings with light and dark stripes that alternate position in time) are nonlinear, invariant to the grating's relative position (or phase) over the dendritic field, so they respond to every reversal (Fig. 1Bii). This nonlinear response called "frequency doubling" reflects the activation of alternating subsets of a Y-cell's bipolar cell inputs (Demb et al., 1999). Because bipolar cells have narrow dendritic (receptive) fields, frequencydoubled responses are elicited preferentially at high spatial frequencies (Fig. $1 A, C$ ). Frequency doubling is the principal physiological property defining Y-cells.

Parasol cells of the primate retina have many properties in common with Y-cells (Crook et al., 2008a). Morphologically, they have large somas, thick axons, and wide dendritic fields. Anatomically, parasol cells project to the magnocellular layers of the LGN. Both cell types are coupled to neighboring cells of the same kind and receive similar patterns of input from interneurons called amacrine cells. Physiologically, parasol cells also have large center-surround receptive fields with transient light responses and form $\mathrm{ON}$ and OFF populations whose dendrites stratify in separate levels near the center of the IPL. Both cell types are sensitive to low contrasts (small deviations from background light level), have high temporal sensitivities, and fast axonal conduction velocities.

Although the similarities listed above are suggestive of homology, parasol cells 

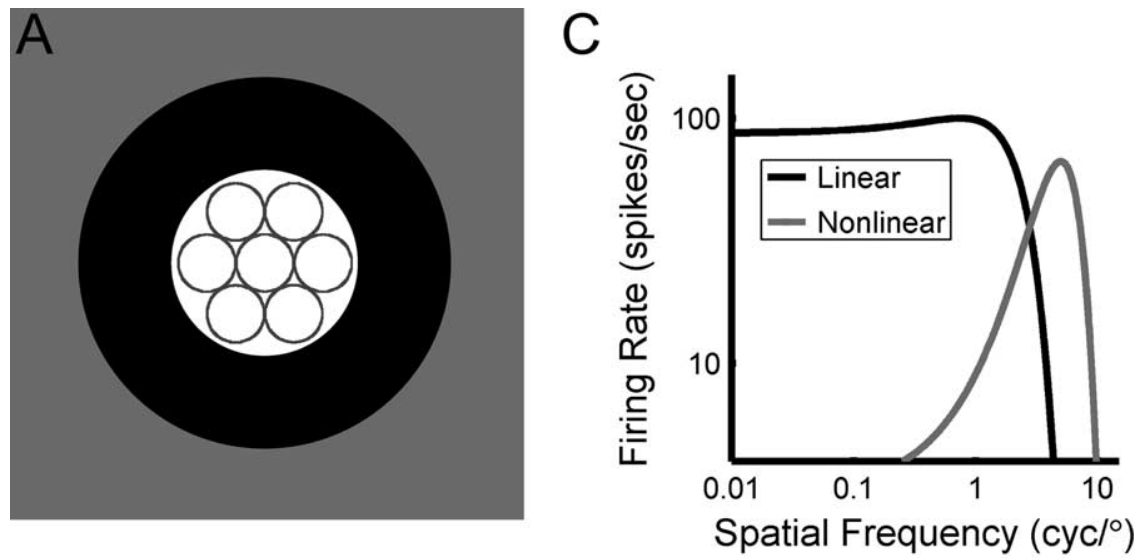

B
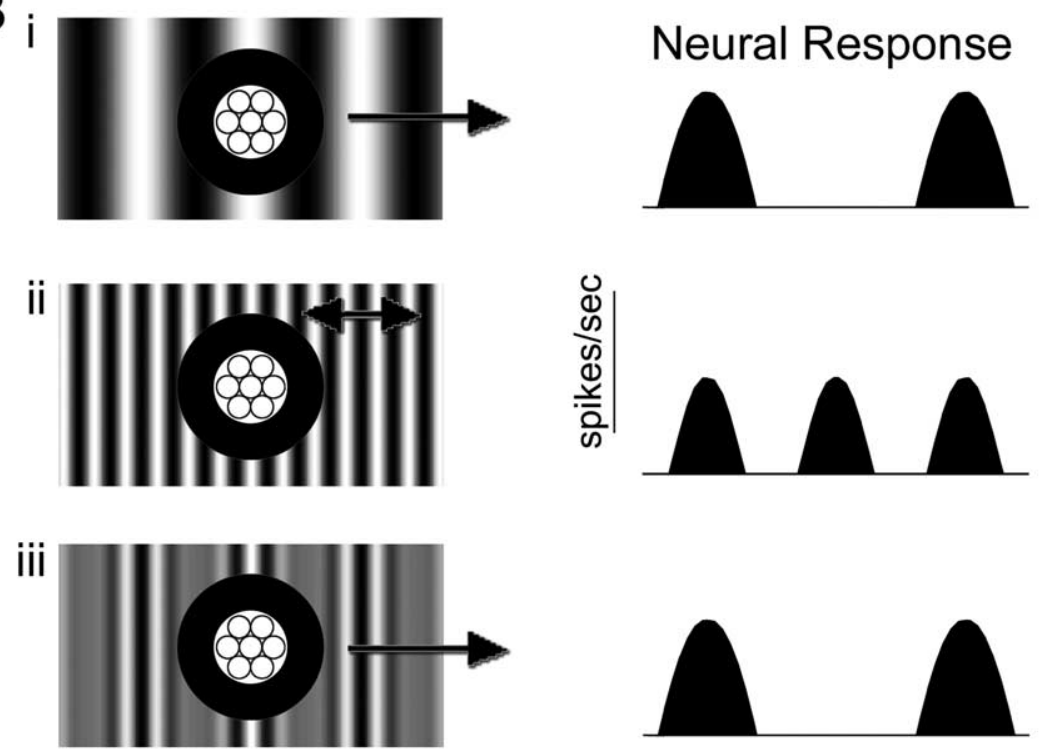

Stimulus:

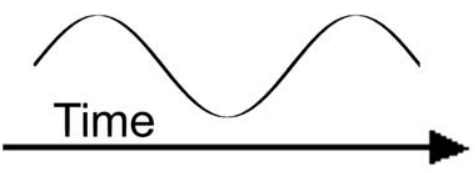

Figure 1. Schematic Y-like receptive field and physiological responses. $A, A n 0 N$ cell responds when the center (white circular region) is stimulated with light brighter than the background (gray square region) or when the surround (black annular region) is stimulated with light that is dimmer. Circles within the center correspond to the receptive fields of bipolar cell inputs. $\boldsymbol{B i}$, An ON Y-cell responds when a bright stripe of a low spatial frequency drifting grating passes over its center. Bii, High spatial frequency contrast-reversing gratings elicit responses at every reversal. Biii, A second-order drifting grating (a high spatial frequency grating whose contrast is modulated at a low spatial frequency) elicits responses when a high contrast region overlies the receptive field center (Demb et al., 2001; Rosenberg et al., 2008). Single-headed arrows over the stimuli indicate drift and the double-headed arrow indicates contrast reversal. $C$, Linear and nonlinear spatial frequency tuning curves similar to those of a parasol cell are represented by two Gaussians.

are thought to lack two defining Y-cell properties: (1) a projection to the superior colliculus and (2) the frequency-doubling nonlinearity. These properties were reinvestigated in Crook et al. (2008a).

Injections of the retrograde tracer rhodamine dextran were made into the primate superior colliculus to establish if parasol cells project to this area. Histologically processed retinas consistently showed large clusters of morphologically identified parasol cells labeled with tracer.
This suggests that as a population, parasol cells do project to the superior colliculus (Crook et al., 2008a).

The frequency-doubling nonlinearity was examined by recording extracellularly from parasol cells in vitro. Consistent with previous findings, low spatial frequency drifting gratings elicited linear responses. Unexpectedly, when contrast-reversing gratings were presented at high spatial frequencies, every recorded parasol cell displayed the frequency-doubled responses characteristic of Y-cells. These nonlinear responses originated from within the receptive field center, where the strongest Y-cell frequency-doubled responses are elicited (Demb et al., 1999; Crook et al., 2008a).

In part, earlier studies conducted in anesthetized preparations likely failed to consistently observe frequency doubling because of difficulties in optimizing the focus of stimuli on the retina. Poor image focus degrades the presentation quality of the high spatial frequencies at which frequency doubling is elicited, and as a result, the magnitude of these nonlinear responses is reduced. By recording in vitro, Crook et al. (2008a) could focus stimuli more precisely, thereby eliminating this problem. In addition, the in vitro retina is not continuously subjected to the hyperpolarizing effect of anesthesia (Nicoll and Madison, 1982). Hyperpolarization may have a more deleterious effect on Y-like nonlinear responses than linear responses because they are typically weaker (Demb et al., 1999, 2001; Rosenberg et al., 2008).

Retinal ganglion cells are defined by a collection of properties. When compared along one or a few dimensions, distinct ganglion cell types can appear similar. For example, in the cat retina, "nonlinear W-cells" display frequency doubling and have wide dendritic fields but are distinguished from Y-cells by their smaller somas and thinner axons (Stone, 1983; Crook et al., 2008a). The primate retina also possesses a second Y-like RGC, the smooth monostratified (SM) cell (Crook et al., 2008b). SM cells, which may be the primate "upsilon cell" identified by Petrusca et al. (2007), can be distinguished from parasol cells morphologically (Crook et al., 2008b). The dendritic fields of SM cells are more sparsely branched and approximately two times wider than those of parasol cells. SM cell somas are also slightly smaller and their axons approximately half as wide. Anatomically, SM cells project to both the LGN and the superior colliculus (Crook et al., 2008b). Although morphologically different, SM and parasol cell dendritic fields are precisely costratified, suggesting that they may share a common input. Consistent with this, physiologically, both cell types have large centersurround receptive fields, form $\mathrm{ON}$ and OFF populations with transient light responses, are sensitive to low contrasts, have high temporal sensitivities, and sum L- and $\mathrm{M}$-cone inputs nonselectively but lack a measurable S-cone input. Most importantly, SM cells display characteristic Y-like 
frequency-doubled responses (Crook et al., 2008b).

The existence of distinct types of Y-like primate RGCs suggests that there may be greater similarities between the early visual systems of primates and other mammals than previously thought. Although it has been suggested that primates lack Y-cells, the findings of Crook et al. $(2008 \mathrm{a}, \mathrm{b})$ led the authors to conclude that parasol and Y-cells are homologous. Because they are morphologically distinguishable from parasol cells and form their own retinal mosaic, SM cells must constitute a second Y-like pathway, perhaps similar to the nonlinear $\mathrm{W}$-cells of the cat retina.

Despite the classification of Crook et al. (2008a,b), it is not entirely clear which of the Y-like primate RGCs is the true homolog to the Y-cell. Homology between Y-cells and parasol (as opposed to SM) cells was argued mainly because parasol cells have larger somas and thicker axons than SM cells. However, the somas of these cell types are only slightly different in size, and although parasol cell axons are thicker, the axons of SM cells are relatively thick compared with many other RGC types. In addition, $\mathrm{W}$-cells in the cat tend to have narrower dendritic fields than Y-cells, but SM cells in the primate have wider dendritic fields than parasol cells (Stone, 1983; Crook et al., 2008a,b). Cat Y-cells and primate SM cells also represent more similar percentages of the total number of RGCs (each $\sim 3 \%$ ), whereas parasol cells represent a larger percentage $(\sim 8 \%)$ (Wässle et al., 1975; Crook et al., 2008b). To establish homology more conclusively, it may be necessary to first develop molecular markers specific to retinal ganglion Y-cells.

The role of Y-like pathways in visual processing is an important area for future research. Recent work has suggested that the Y-cell frequency-doubling nonlinearity is important for detecting "second-order" features in which there is variation in the contrast of a fine-grained pattern but no variation in mean luminance (Demb et al., 2001; Rosenberg et al., 2008). Although not yet investigated, parasol and SM cells may serve this function within the primate visual system. If this is the case, a selective loss of their nonlinear responses in anesthetized preparations may help to explain discrepancies between second-order motion studies conducted in primate area MT (Albright, 1992; O'Keefe and Movshon, 1998). The functional significance of having multiple Y-like pathways is also of interest. Because parasol and SM cells have different dendritic (receptive) field sizes, the transformations they perform on a visual scene operate on separate spatial scales (Crook et al., 2008a,b). There may also be important functional distinctions between these cell types that have not yet been identified. In the cat LGN, for example, the presentation of second-order stimuli reveals systematic variability in the nonlinear tuning properties of Y-cells that cannot be predicted from responses to simpler stimuli (Rosenberg et al., 2008). Most importantly, the results of Crook et al. (2008a,b) require that our understanding of the role that the primate magnocellular pathway plays in visual processing be revised to incorporate the nonlinear Y-like properties of parasol and SM cells.

\section{References}

Albright TD (1992) Form-cue invariant motion processing in primate visual cortex. Science 255:1141-1143.

Crook JD, Peterson BB, Packer OS, Robinson FR, Troy JB, Dacey DM (2008a) Y-cell receptive field and collicular projection of parasol gan- glion cells in macaque monkey retina. J Neurosci 28:11277-11291.

Crook JD, Peterson BB, Packer OS, Robinson FR, Gamlin PD, Troy JB, Dacey DM (2008b) The smooth monostratified ganglion cell: evidence for spatial diversity in the Y-cell pathway to the lateral geniculate nucleus and superior colliculus in the macaque monkey. J Neurosci 28:12654-12671.

Demb JB, Haarsma L, Freed MA, Sterling P (1999) Functional circuitry of the retinal ganglion cell's nonlinear receptive field. J Neurosci 19:9756-9767.

Demb JB, Zaghloul K, Sterling P (2001) Cellular basis for the response to second-order motion cues in $\mathrm{Y}$ retinal ganglion cells. Neuron 32:711-721.

Enroth-Cugell C, Robson JG (1966) The contrast sensitivity of retinal ganglion cells of the cat. J Physiol 187:517-552.

Nicoll RA, Madison DV (1982) General anesthetics hyperpolarize neurons in the vertebrate central nervous system. Science 217:1055-1057.

O'Keefe LP, Movshon JA (1998) Processing of first- and second-order motion signals by neurons in area MT of the macaque monkey. Vis Neurosci 15:305-317.

Peichl L, Ott H, Boycott BB (1987) Alpha ganglion cells in mammalian retinae. Proc R Soc Lond B Biol Sci 231:169-197.

Petrusca D, Grivich MI, Sher A, Field GD, Gauthier JL, Greschner M, Shlens J, Chichilnisky EJ, Litke AM (2007) Identification and characterization of a Y-like primate retinal ganglion cell type. J Neurosci 27:11019-11027.

Rosenberg A, Husson TR, Mallik AK, Issa NP (2008) Frequency-doubling in the early visual system underlies sensitivity to secondorder stimuli. J Vis 8:281a.

Stone J (1983) Parallel processing in the visual system: the classification of retinal ganglion cells and its impact on the neurobiology of vision. New York: Springer.

Wässle H, Levick WR, Cleland BG (1975) The distribution of the alpha type of ganglion cells in the cat's retina. J Comp Neurol 159:419438. 